\title{
Testing the evolution of the DB white dwarf GD 358: first results of a new approach using asteroseismology
}

\author{
J. M. González Pérez ${ }^{1}$ and T. S. Metcalfe ${ }^{2}$
}

\author{
${ }^{1}$ Instituto de Astrofísica de Canarias, 38200 La Laguna, Tenerife, Spain \\ e-mail: jgperez@iac.es \\ ${ }^{2}$ High Altitude Observatory, National Center for Atmospheric Research, PO Box 3000, Boulder, Colorado, USA
}

Received 14 February 2008 / Accepted 3 October 2008

ABSTRACT

\begin{abstract}
Aims. We present a new method that investigates the evolutionary history of the pulsating DB white dwarf GD 358 using asteroseismology. This is done considering the internal $\mathrm{C} / \mathrm{O}$ profile, which describes the relative abundances of carbon and oxygen from the core of the star to its surface. Different evolutionary channels lead to the generation of different $\mathrm{C} / \mathrm{O}$ profiles, and these affect the pulsation periods.

Methods. We used the $\mathrm{C} / \mathrm{O}$ profiles associated with white dwarfs that evolved through binary evolution channels where the progenitor experienced one or two episodes of mass loss during one or two common envelope (CE) phases, and two profiles from single-star evolution. We computed models using these different profiles and used a genetic algorithm (GA) to optimize the search in the parameter space for the best fit to the observed pulsation periods. We used three-parameter models, adjusting the stellar mass $\left(M_{\star}\right)$, the effective temperature $\left(T_{\mathrm{eff}}\right)$, and the helium mass of the external layer $\left(M_{\mathrm{He}}\right)$.

Results. Our results suggest that binary evolution profiles may provide a better match to the pulsation periods of GD 358 . The best fit to the observations is obtained using a profile related to an evolutionary history where two episodes of mass loss happen during two CE phases, the first during the RGB (red giant branch) stage. The values obtained are $T_{\text {eff }}=24300 \mathrm{~K}, M_{\star}=0.585 M_{\odot}$, and $\log \left(M_{\mathrm{He}} / M_{\star}\right)=-5.66$. The best-fit model has a mass close to the mean mass for DB white dwarfs found in various works and a temperature consistent with UV spectra obtained with the IUE satellite.
\end{abstract}

Key words. stars: oscillations - stars: white dwarfs - stars: individual: GD 358 - stars: evolution - stars: interiors

\section{Introduction}

Pulsating stars are found among white dwarfs. Almost every star in our Galaxy will eventually become a white dwarf. Since they are relatively simple compared to main sequence stars, white dwarfs provide one of the best opportunities for learning about stellar structure and evolution. Their interior structure contains a record of the physical processes that operate during the later stages in the lives of most stars, so there is a potential amount of information encoded in their pulsation frequencies. The observational requirements of white dwarfs asteroseismology have been addressed by the development of the Whole Earth Telescope (Nather et al. 1990).

There are presently three known classes of pulsating white dwarfs ${ }^{1}$. The hottest class is the pulsating PG 1159 stars (also known as DOV stars), which are hydrogen-deficient stars with surface layers rich in helium, carbon, and oxygen. Some of them are still embedded in a planetary nebula and are commonly called PNNVs. PNNVs show complicated and variable temporal spectra (Gonzalez Perez et al. 2006). PG 1159 stars require detailed calculations that evolve a main sequence stellar model to the pre-white dwarf phase to yield accurate pulsation periods (e.g. Corsico et al. 2008). The two cooler classes are the heliumatmosphere variable (DBV) and hydrogen-atmosphere variable (DAV) white dwarfs. The pulsation periods for these objects can

1 The recent discovery of pulsations in the hot DQ star SDSS J142 625.71+575218.3 could set it as the first member of a new class of pulsating white dwarfs (Montgomery et al. 2008). be calculated accurately by evolving simpler, less detailed models. The DAV stars are generally modeled with a core of carbon and oxygen with an overlying blanket of helium covered by a thin layer of hydrogen on the surface. The DBV stars are the simplest of all, with no detectable hydrogen and only a helium layer surrounding the carbon/oxygen core. Robinson et al. (1982) and Kepler (1984) demonstrated that the variable white dwarf stars pulsate in non-radial gravity modes.

The chemical evolution of the DB white dwarfs atmospheres cannot be satisfactorily explained to date (see Shipman 1997, for a review). In particular, the presence of the DB gap, which is the absence of DB white dwarfs between temperatures of 30000-45000 K (e.g. Liebert 1986), is poorly understood. This DB gap persists in the latest results from the Sloan Digital Sky Survey (Kleinman et al. 2004). Eisenstein et al. (2006) found several DBs in the gap, but still a decrease in the relative number. One possibility is that DBs might turn into DAs (white dwarfs with pure $\mathrm{H}$ atmospheres) through diffusion of small traces of $\mathrm{H}$ to the surface of the star, concealing the $\mathrm{He}$ atmosphere. However, Castanheira et al. (2006) showed that atmospheric contamination with $\mathrm{H}$ is not directly proportional to $T_{\text {eff }}$ for DB stars, in conflict with what would be expected under the diffusion scenario.

It is also not clear whether DBs are mostly produced by single-star evolution or whether a significant fraction of them originate from binary progenitors. Nather et al. (1981) pointed out that interacting binary white dwarfs (IBWDs) should produce DBs at the end of their evolution. AM CVn, one of the best 
studied IBWDs, has an $T_{\text {eff }} \sim 25000 \mathrm{~K}$. This temperature is inside the interval where DBVs are found. If AM CVn ends mass transfer phase at this temperature, there will be a single DB left, quite possibly a DBV. The examination of these problems can be aided by asteroseismology. Compared with other classes of pulsating stars, the DBVs are promising candidates for asteroseismology: their mode spectra are relatively rich, and pulsation theory for these stars is quite advanced and well tested.

GD 358, also called V777 Herculis, is the prototype of the DBVs and is one of the classical examples of the application of asteroseismological methods (e.g. Winget et al. 1994; Vuille et al. 2000; Kepler et al. 2003). GD 358 was the first pulsating star detected based on a theoretical prediction (Winget et al. 1985). When the Whole Earth Telescope observed GD 358 in 1990, 181 periodicities were detected, but only modes from radial order $k=8$ to 18 were identified, most of them showing triplets, consistent with the degree $l=1$ identification (Winget et al. 1994). Detecting as many modes as possible is important, as each independent mode detected yields an independent constraint on the star's structure. The authors derived the total stellar mass as $0.61 \pm 0.03 M_{\odot}$, the mass of the outer helium envelope as $(2.0 \pm 1.0) \times 10^{-6} M_{\star}$, the luminosity as $0.050 \pm 0.012 L_{\odot}$ and, deriving a temperature and bolometric correction, the distance as $42 \pm 3$ pc.

Asteroseismological analysis of GD 358 has been a successful tool to test pulsation theory. For instance, the central oxygen abundance of the models has been shown to have a measurable effect on the pulsation frequencies (Metcalfe et al. 2001, hereafter M1), and also the possible presence of a ${ }^{3} \mathrm{He} /{ }^{4} \mathrm{He}$ transition zone caused by chemical diffusion (Montgomery et al. 2001), although more recent observations have ruled out this possibility (Wolff et al. 2002).

This article is organised as follows: in Sect. 2 we introduce the $\mathrm{C} / \mathrm{O}$ profiles and the dependence of the pulsation pattern with their shape. In Sect. 3 we briefly introduce and describe the close evolutionary channels that are used in this study. In Sect. 4 we show how the computation of models is done, including an overview of the DB white dwarf models, the genetic algorithm used like tool to improve the effectiveness to look for the best-fit model, a justification of the parameters space used, and a description about how the profiles are computed. Finally, in Sects. 5 and 6 we present and discuss the results.

\section{Pulsations and $\mathrm{C} / \mathrm{O}$ profiles}

The interior structure of the white dwarfs contains a record of the physical processes that operate during the later stages in the lives of most stars. The distribution of the $\mathrm{C} / \mathrm{O}$ ratio depends on the evolution of the star. Stellar evolution theory predicts that a large number of white dwarfs are post Asymptotic Giant Brach (AGB) stars. Therefore, they should consist of the primary ashes of He-burning, i.e. carbon and oxygen. The central ratio $\mathrm{C} / \mathrm{O}$ depends on the interplay between the triple- $\alpha$ and the ${ }^{12} \mathrm{C}(\alpha, \gamma){ }^{16} \mathrm{O}$ reactions during the core helium burning phase. In the first part of the burning the most efficient process is the triple- $\alpha$, which produces ${ }^{12} \mathrm{C}$. When the central abundance of helium decreases below about $0.15 M_{\star}$, the production of ${ }^{16} \mathrm{O}$ via the ${ }^{12} C(\alpha, \gamma){ }^{16} \mathrm{O}$ becomes the dominant process.

While they are still embedded in the cores of red giant models, the internal chemical profiles of white dwarfs models show a relatively constant $\mathrm{C} / \mathrm{O}$ ratio near the center, with a size determined by the extent of the helium-burning convective region. Further out the oxygen mass fraction decreases as a result of the helium-burning shell moving towards the surface of the red giant model while gravitational contraction causes the temperature and density to rise. This increases the efficiency of the triple- $\alpha$ reaction, producing more carbon relative to oxygen. The central oxygen mass fraction is lower in higher mass white dwarfs models. The rate of the triple- $\alpha$ reaction increases faster at higher densities than does the ${ }^{12} \mathrm{C}(\alpha, \gamma){ }^{16} \mathrm{O}$ reaction. As a consequence, more helium is used up in the production of carbon, and relatively less is available to produce oxygen in higher mass models.

Metcalfe et al. (2000, hereafter M0) used the observed periods of GD 358 from Winget et al. (1994) to search for the optimum theoretical model with static diffusion envelopes. This search was based in the application of a genetic-algorithm-based optimization (GA) to white dwarf pulsation models, which performs a global search to provide objective global best-fit models for the observed pulsation frequencies. They used a threeparameter model including $M_{\star}, T_{\mathrm{eff}}$, and $M_{\mathrm{He}}$, and six different combinations of core composition and $\mathrm{C} / \mathrm{O}$ transition profiles to search for the best-fit model. Their results showed that both the central oxygen abundance and the shape of the $\mathrm{C} / \mathrm{O}$ profile affect the pulsation pattern.

M1 modified the code to include as free parameters any central oxygen mass fraction $\left(X_{0}\right)$ between 0 and 1 with resolution $1 \%$ and a fractional mass parameter $(q)$. The GA fitting process explored different chemical profiles built in the following way: the value $X_{0}$ was fixed to its central value out to $q$ that varied between 0.1 and 0.85 with resolution $0.75 \%$. From this point $X_{0}$ decreased linearly in mass to zero oxygen at the $95 \%$ mass point. The results showed that significant improvement to the fit was possible by including $X_{0}$ and $q$ as free parameters, confirming that the observed pulsations really contain information about the hidden interiors of the stars. The best fit obtained was: $T_{\text {eff }}=22600 \mathrm{~K} ; M_{\star}=0.650 M_{\odot} ; \log \left(M_{\mathrm{He}} / M_{\star}\right)=-2.74$; $X_{0}=0.84 ; q=0.49$.

Therefore, the $\mathrm{C} / \mathrm{O}$ profiles may give us an opportunity to explore the previous evolution of the DBV white dwarfs. C/O profiles obtained from single evolutionary models are different than those obtained from evolutionary models of close binary systems (e.g. Iben \& Tutukov 1985, hereafter IT85; Iben 1986). The shapes of the $\mathrm{C} / \mathrm{O}$ profiles may be affected due to interaction with close companions, i.e., if $\mathrm{CE}$ phases happen during the RGB and/or AGB stages.

\section{Close binary evolution}

Peculiar white dwarf stars may be created through binary evolutionary channels. We have concentrated on evolutionary channels that produce white dwarfs without hydrogen. Hydrogen may be removed of the system due to previous CE stages. The resulting white dwarfs may have a surface rich in helium and may contribute to the DB population.

IT85 analyzed the possibility to obtain such white dwarfs by evolving components of close binary systems where the component masses were in the range 2 to $12 M_{\odot}$. They assumed models where the more massive component filled its Roche lobe during the RGB, after the central hydrogen exhaustion, but before or near the onset of core helium burning. The binary components experience one or two mass loss processes due to CE stages, depending upon the initial main-sequence mass.

Components with masses in the range 2.3 to $4.8 M_{\odot}$ experience one episode of mass loss and become degenerate dwarfs of mass in the range 0.32 to about $0.7 M_{\odot}$. The remnants experience several small hydrogen shell flashes and one large final flash which, for the more massive degenerates dwarfs, 
remove almost all of the hydrogen-rich layer. In these cases the total mass of the hydrogen layer is reduced to about $10^{-5} M_{\odot}$. This evolutionary channel may produce remnants with masses as small as $0.4 M_{\odot}$ and can convert $70 \%$ of the initial helium into carbon/oxygen. This represent a substantial difference from evolutionary channels of single low-mass stars, which do not ignite and burn helium until the mass of the electron-degenerate cores reaches $\sim 0.5 M_{\odot}$. Han et al. (2000) have performed close binary evolutionary calculations and found that a carbon/oxygen white dwarf with mass as low as $0.33 M_{\odot}$ may be formed from stable Roche overflow if the initial mass of the primary is close to $2.5 M_{\odot}$.

IT85 found that binary components of initial mass in the range 4.8 to $10.3 M_{\odot}$ lose the hydrogen-rich matter in two episodes of Roche-lobe overflow and become $\mathrm{C} / \mathrm{O}$ degenerate dwarfs with masses in the range 0.7 to $1.08 M_{\odot}$. This channel leads to the production of DB white dwarfs. For the more massive remnants (over $0.8 M_{\odot}$ ), a substantial part of the helium layer is also removed from the system during the second Rochelobe overflow, so the degenerate dwarf would have a thin helium outer layer. This layer is expected to be thicker for remnants below $0.8 M_{\odot}$. IT85 estimated a formation rate of $\sim 10^{-2} \mathrm{yr}^{-1}$ of non-DA degenerate dwarfs created for these binary scenarios and concluded that they may produce $15 \%-20 \%$ of all hot non-DA white dwarfs.

\section{Computation of models}

\subsection{DB white dwarfs models}

To find the theoretical model of a white dwarf, we start with a static model of a pre-white dwarf and allow it to evolve quasistatically until it reaches the desired temperature. We then calculate the adiabatic non-radial oscillation frequencies for the output model. It is not necessary to go through detailed calculations that evolve a main sequence star all the way to its pre-white dwarf phase. For the DB and DA white dwarfs, it is sufficient to start with a hot polytrope of order $2 / 3$ (i.e. $P \propto \rho^{5 / 3}$ ). The cooling tracks of these polytropes converge with those of the pre-white dwarfs models above the temperatures at which DB and DA white dwarfs are observed to be pulsationally unstable (Wood 1990).

We have used the White Dwarf Evolution Code (WDEC) to evolve a start model to a specific temperature. This code is described in detail by Lamb \& Van Horn (1975) and by Wood (1990). It was originally written by Martin Schwarzschild, and has subsequently been updated and modified by many others including: Kutter \& Savedoff (1969), Lamb \& Van Horn (1975), Winget (1981), Kawaler (1986), Wood (1990), Bradley (1993), and Montgomery (1998). The pulsation frequencies of the output models are determined using the adiabatic non-radial oscillation (ANRO) code described by Kawaler (1986). Practical modifications to these programs were done by $\mathrm{M} 0$, primarily to allow models to be calculated without any intervention by the user.

The equation of state for $\mathrm{H} / \mathrm{He}$ comes from Saumon et al. (1995). We use the OPAL opacity tables from Iglesias \& Rogers (1996), neutrino rates from Itoh et al. (1996), and the ML2 mixing-length prescription of Böhm \& Cassinelli (1971). We have fixed the ratio of mixing-length/pressure scale height to 1.25 , as recommended by Beauchamp et al. (1999). The evolution calculations for the core are fully self-consistent, but the envelope is treated separately. We have employed a single layered configuration. Montgomery et al. (2003) showed that there is an inherent symmetry in the sensitivity of the pulsations to structure in the core and in the envelope, and at the temperature of GD 358 this makes it essentially impossible to probe both structures at the same time. They also found that core structure is more important to determine the pulsation periods than the outer helium layer. The core and envelope are stitched together and the envelope is adjusted to match the boundary conditions at the interface. Adjusting the helium layer mass involves stitching an envelope with the desired thickness onto the core before starting the evolution. Because this is done while the model is still very hot, there is plenty of time to reach equilibrium before the model approaches the final temperature.

\subsection{The genetic algorithm and Darwin}

The global search for the optimal model parameters to fit the independent pulsations periods of GD 358 is done using the GA optimization method developed by M0 and M1. This method improves the objective search in the parameter space over traditional procedures like iterative methods starting from a first guess. This standard approach has a potential problem: the initial set of parameters is usually determined subjectively. This, combined with a local search in the parameter space, may produce a local good fit that is not the global best fit. Restrictions by the GA method regarding the range of the parameters space are imposed only by observational constraints and the physics of the model. Therefore, GA provides a relatively efficient way of searching globally for the best-fit model.

The first step of the GA is to fill up the parameter space with trials consisting of randomly chosen values for each parameter, within a range based on the physics that the parameters are supposed to describe. The model is evaluated for each trial, and the result is compared with the observational data. This comparison assigns a fitness to the trial inversely proportional to the variance. The new generation of trials is then created taking into account the previous fitness. The fittest models survive to the next generation and the parameter space is then sampled more around these values. Operators emulating reproduction and mutation are applied to produce the new generation of trials. The evolution continues for a specified number of generations, chosen to maximize the efficiency of the method. We used a population size of 128 trials and allowed the GA to run for 200 generations. We have performed a total of $10 \mathrm{GA}$ runs for each core composition to reduce the chances of not finding the best answer to less than about 3 in 10000 .

The GA code has been implemented in a network of 64 PCs running Linux (Metcalfe \& Nather 1999). The white dwarf code runs on this metacomputer by using the Parallel Virtual Machine (PVM) software (Geist et al. 1994), which allows a collection of networked computers to cooperate on a problem as if they were a single multiprocessor parallel machine. The central computer in the network is called Darwin and runs the parallel version of the genetic algorithm (PIKAIA) which is the master program. After creating a new generation, Darwin distributes to the slave computers an array with the models to check. The slave computers perform the following actions: evolve a white dwarf model to the specified temperature, determine the pulsation periods of the model, and compare observations with the calculated pulsation periods. Then it sends the result back to Darwin which will include it in the GA code. The slave computer is then ready for a new trial so Darwin can send a new trial from the generation. 


\subsection{Parameter space}

We use a three-parameter model including $M_{\star}, T_{\text {eff }}$, and $M_{\mathrm{He}}$. The GA search space is defined as follows: the masses are confined between $0.45 M_{\odot}$ and $0.95 M_{\odot}$. Although Kepler et al. (2007) reported a substantial number of white dwarfs over this upper limit, Beauchamp et al. (1999) and Castanheira et al. (2006) found that all known DBVs appear to have masses within this range. The temperature search includes values between $20000 \mathrm{~K}$ and $30000 \mathrm{~K}$ and is based in the temperature determination for the known DB instability strip, that is, the strip in the $T_{\text {eff }}$ versus $\log (g)$ diagram where the DB white dwarfs are pulsationally unstable (Beauchamp et al. 1999). These authors, depending on various assumptions, place the red edge as low as $21800 \mathrm{~K}$, and the blue edge as high as $27800 \mathrm{~K}$. Finally, the search interval for the mass of the atmospheric helium layer has a lower limit $10^{-8} M_{\star}$ and an upper limit $10^{-2} M_{\star}$. Masses must not be greater than this because the pressure on the overlying material will then theoretically initiate helium burning at the base of the envelope. At the other extreme, none of the models pulsate for helium masses less than $10^{-8} M_{\star}$ over the entire temperature range we consider (Bradley \& Winget 1994).

A two-digit decimal is used for encoding the different parameters. This results in a temperature resolution of $100 \mathrm{~K}$, a mass resolution of $0.005 M_{\odot}$, and a resolution for the helium layer thickness of 0.05 dex.

\subsection{Computing profiles}

We have used the $\mathrm{C} / \mathrm{O}$ profiles presented in IT85 and Iben (1986). We had to modify the original profiles to be able to incorporate them into our code. The axes have been transformed to $X_{\mathrm{O}}$ (mass fraction of oxygen) versus $M_{r} / M_{\star}$ (enclosed mass fraction at a given radius). A $\mathrm{C} / \mathrm{O}$ profile is computed using a series of pairs of points $\left(X_{\mathrm{C}}, M_{r} / M_{\star}\right)$, where $X_{\mathrm{C}}=1-X_{\mathrm{O}}$. These pairs define the profile shape in the model. We have used between 6 and 8 points. This procedure simplifies the original shape of the profile but reproduces it well. M1 have shown that the employment of this kind of simplification do not affect significantly the resulting period fits. Their Fig. 5, which shows the bumps in the Brunt-Väisälä curve from simple profiles likes the ones used in this work, and from the detailed profile of Salaris et al. (1997), demonstrates that the simple profiles do not produce spikes any larger than the detailed profiles. The reason is that the code automatically smooths the transitions by adjusting the spacing of the interior model shells to avoid such unphysical spikes.

One limitation for computing the profiles is that $X_{\mathrm{O}}$ must be set to zero outside the mass fraction $\left(M_{r} / M\right)=0.95$. However, some of the original profiles present some amount of oxygen outside this fractional mass. Another simplification is that our models consider carbon and oxygen as the only nuclear species in the interiors of the white dwarfs. IT85 evolutionary models present traces of other elements like ${ }^{22} \mathrm{Ne}$ and ${ }^{25} \mathrm{Mg}$. Figures 1 and 2 show the profiles we tested.

Our study is mainly based on IT85 because, until now, it is the only work presenting several $\mathrm{C} / \mathrm{O}$ profiles for different evolutionary channels of close binary systems. In addition, the profiles are obtained using the same physics, which improve the possibilities for a quantitative comparison. Other profiles for single evolutionary scenarios are available (e.g. Salaris et al. 1997). However, the physics included in these evolutionary models are slightly different, e.g. how overshooting is treated, nuclear reactions rates, etc. Models with different physics may produce different $\mathrm{C} / \mathrm{O}$ profile shapes.
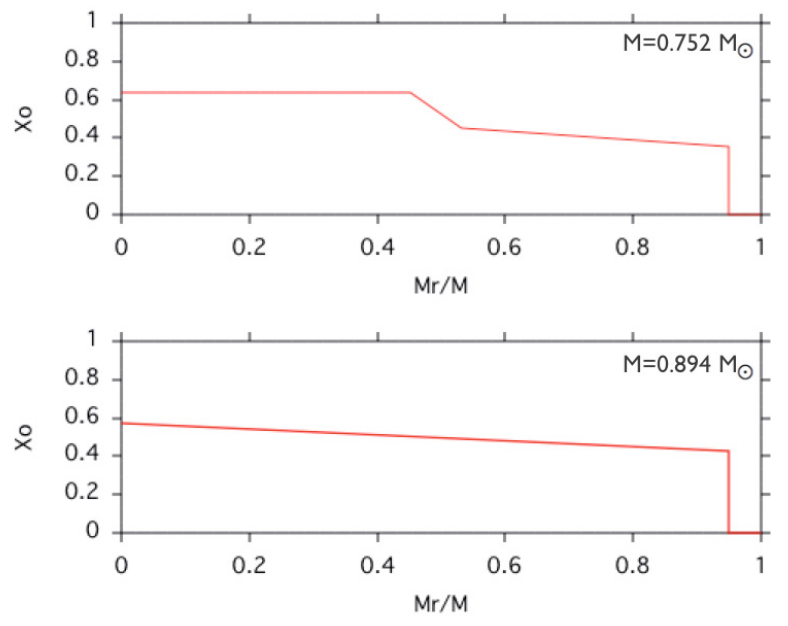

Fig. 1. Profiles of evolutionary channels affected by 2 epochs of mass loss during $2 \mathrm{CE}$ phases, the first one during the RGB stage. The upper panel shows the simplified profile associated with the original chemical profile shown in Fig. 12 of IT85, and obtained for a $0.752 M_{\odot}$ remnant. The lower panel corresponds to Fig. 13 of IT85 and is obtained for a $0.894 M_{\odot}$ remnant.

Different scenarios happen during the single and binary channels that lead to differences in the resulting $\mathrm{C} / \mathrm{O}$ profiles. For instance, IT85 show that their lowest mass helium-burning remnant has a long lifetime compared with the single-star counterpart, and that the average temperatures and densities, which will affect to the $\mathrm{C} / \mathrm{O}$ ratio, is lower for the single-star counterpart. This is consequence of the absence of a large reservoir of hydrogen due to the mass loss during the first common envelope episode, and the consequent approximate constancy of the mass of the hydrogen-exhausted core. This can be seen when comparing the lower panel in Fig. 1 with the lower panel in Fig. 2. These two profiles are adequate for this comparison because they have close initial masses, being one related to binary evolution and the other to single evolution. In the first case, the central oxygen fraction is, as expected, lower. This behavior became less important as the mass of the star increases, because the amount of matter added to the hydrogen-burning phase becomes a smaller fraction of the core mass.

IT85 also show that for the same progenitor main-sequence star mass, during the shell helium-burning phase, the contracting $\mathrm{CO}$ in a close binary component, when it fills the Roche lobe for second time, is smaller than that of a single-star counterpart during the AGB stage. Therefore, the maximum interior temperature is smaller for the close binary component. During this stage, the oxygen fraction is higher within the central region defined by the convective core during the latter stages of core helium burning than is in the shell region which has been processed through shell helium burning. Thus, the $\mathrm{C} / \mathrm{O}$ profile shape related to the convective core, where the oxygen fraction is approximate constant in almost all the profiles presented, depends on the evolutionary channel as well. When comparing remnants which have evolved from models with the same initial mass, the oxygen mass fraction is expected to be more significant in those evolved through single evolutionary channels. Finally, the first ramp that appears in many $\mathrm{C} / \mathrm{O}$ profiles is smoother in binary evolution remnants because they avoid instabilities that occur in AGB stars, which cause this feature. The main reason is because the effective accretion rate matter during the shell burning 

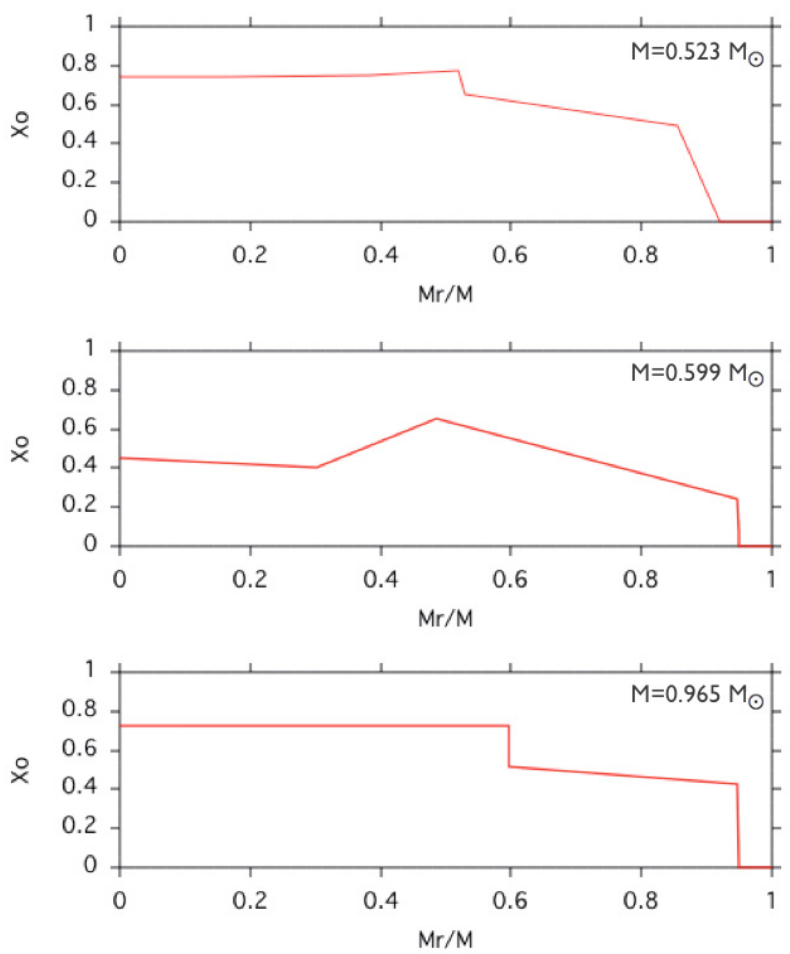

Fig. 2. Profiles of an evolutionary channel affected by $1 \mathrm{CE}$, and 2 evolutionary channels of single stars. The upper panel is the simplified version of the profile corresponding to Fig. 27 of IT85, obtained for a $0.523 M_{\odot}$ remnant with a previous evolutionary history affected by 1 process of mass loss due to $1 \mathrm{CE}$ during the RGB stage. The middle panel corresponds to Fig. 16 of IT85, and is obtained using a horizontalbranch star of initial mass $7 M_{\odot}$ that produces a remnant of $0.599 M_{\odot}$. The lower panel corresponds to Fig. 15 of IT85 and is related to a white dwarf model of mass $0.965 M_{\odot}$ that evolved from a single AGB model star of mass $7 M_{\odot}$

Table 1. Characteristics of the evolutionary channels of the profiles we tested. The second column indicates how many episodes of mass loss have happened due to $\mathrm{CE}$ phases in the previous evolution. RGB indicates that the first happened during the RGB stage. SE indicates single evolution. HB indicates that the white dwarf evolved from a Horizontal Branch model, and AGB from an Asymptotic Giant Branch model.

\begin{tabular}{llll}
\hline \hline Profile id & Evolution & Mass WD $\left(M_{\odot}\right)$ & Hydrogen \\
\hline 1 & 2 CE, RGB & 0.752 & no \\
2 & 2 CE, RGB & 0.894 & no \\
3 & 1 CE, RGB & 0.523 & $\sim 2.6 \times 10^{-4}$ \\
4 & SE, HB & 0.599 & yes \\
5 & SE, AGB & 0.965 & yes \\
\hline
\end{tabular}

is much larger than that of a AGB star of comparable core mass and luminosity.

IT85 evolutionary models start with an initial pair of stars with certain masses and separation and are evolved to produce a white dwarf with a certain $\mathrm{C} / \mathrm{O}$ profile and mass, mainly without hydrogen on the surface. Therefore, each $\mathrm{C} / \mathrm{O}$ profile tested is already related to a certain mass. In our code, $M_{\star}$ is a free parameter. A self-consistent result for a given profile would require a best-fit model with a value for $M_{\star}$ not too far from the final masses in IT85, though profiles for different masses should be qualitatively similar.
Table 2. Best models obtained for the different evolutionary channels we tested. The fits labeled with " $a$ " are not compatible with the observed parallax of GD 358, which constrains the luminosity. The best-fit model is emphasized with *.

\begin{tabular}{lllll}
\hline \hline Profile id & $T_{\text {eff }}(\mathrm{K})$ & $M / M_{\odot}$ & $\log \left(M_{\mathrm{He}} / M_{\star}\right)$ & rms (s) \\
\hline 1 & 25000 & 0.555 & -5.60 & 2.13 \\
$2^{*}$ & 24300 & 0.585 & -5.66 & 2.12 \\
3 & 23700 & 0.605 & -6.03 & 2.19 \\
4 & 25100 & 0.825 & -3.96 & $2.23^{a}$ \\
5 & 26000 & 0.560 & -5.39 & 2.21 \\
\hline
\end{tabular}

Table 1 presents the properties of the different $\mathrm{C} / \mathrm{O}$ profiles we tested. The second column indicates how many episodes of mass loss have happened due to CE phases in the previous evolution. RGB indicates that the first CE happened during the Red Giant Brach stage. SE indicates single evolution. HB indicates a white dwarf remnant coming from a horizontal branch model, and AGB from an Asymptotic Giant Branch model. We have included two profiles associated with a degenerate white dwarf that has experienced 2 epochs of mass loss in CE episodes, the first one during the RGB phase: profile 1 is from the $0.752 M_{\odot}$ remnant of a model of initial mass $5 M_{\odot}$ (see upper panel of Fig. 1), while profile 2 comes from a $0.894 M_{\odot}$ remnant of a model with initial mass $6.95 M_{\odot}$ (see lower panel of Fig. 1). Profile 3 corresponds to the $0.523 M_{\odot}$ remnant of a model of initial mass $4 M_{\odot}$ that has experienced only one episode of mass loss due to a $\mathrm{CE}$ during the RGB phase. This model retains some hydrogen in the surface layer, $\sim 2.6 \times 10^{-4} M_{\star}$ (see profile in the upper panel of Fig. 2).

We have completed our comparative analysis by checking the best-fit models for 2 profiles obtained from single evolutionary scenarios. Profile 4 is related to a white dwarf model of mass $0.599 M_{\odot}$ that comes from the evolution of a horizontal-branch model of initial mass $7 M_{\odot}$ (Iben 1982). This is presented in the middle panel of Fig. 2). Profile 5 is the remnant $0.965 M_{\odot}$ core of a single AGB model star of mass $7 M_{\odot}$ (Iben 1976, see lower panel of Fig. 2). This last model presents some inconsistency since our parameter space for the mass is limited to masses below $0.95 M_{\odot}$.

\section{Results}

Table 2 presents the best fits obtained for each profile. The best fits are obtained for the profiles 1 and 2, which involve evolutionary channels where the star experiences two CE phases. Profile 1 is associated in the IT85 evolutionary model to a white dwarf with mass $0.752 M_{\odot}$, and profile 2 to a more massive white dwarf $\left(0.894 M_{\odot}\right)$. The asteroseismological masses obtained are in both cases smaller: 0.555 and $0.585 M_{\odot}$. Both fits have almost the same residuals, 2.13 and $2.12 \mathrm{~s}$, but the values obtained for the 3 parameters, $T_{\mathrm{eff}}, M_{\star}$, and $\log \left(M_{\mathrm{He}} / M_{\star}\right)$, are slightly different. This confirms the dependence of the models on the intrinsic shape of the profile.

The best fit is obtained using profile 2 . The parameters obtained are $T_{\text {eff }}=24300 \mathrm{~K}, M_{\star}=0.585 M_{\odot}$, and $\log \left(M_{\mathrm{He}} / M_{\star}\right)=-5.66$. The best-fit model has a mass close to the mean mass for DB white dwarfs, and a temperature consistent with UV spectra obtained with the IUE satellite (Castanheira et al. 2005). This model uses a thin helium envelope, which justifies the use of single layered models. Althaus and Corsico (2004) have shown that for thin helium envelopes with fractional masses between $10^{-4}$ and $10^{-3}$, the double layered structure can evolve 


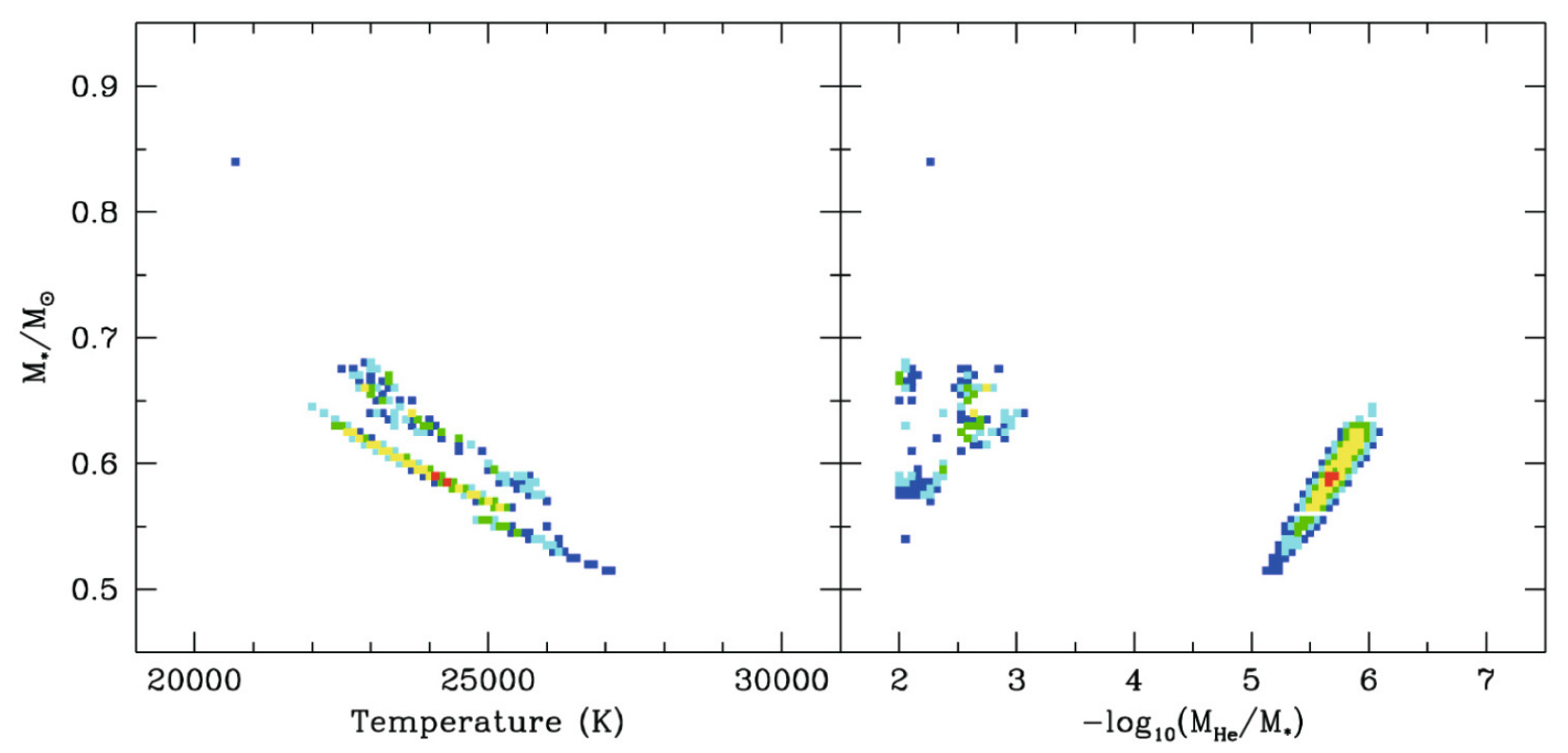

Fig. 3. Front and side views of the search space for the best model (profile 2). Square points mark the location of models that yields a reasonable match to the periods observed for GD 358. The color of a point indicates the relative quality of the match (see text for details).

to a single layered structure near the temperature of GD 358 (their Fig. 5), and this is even more true of higher mass models (their Fig. 8).

Figure 3 shows the general characteristics of the threedimensional parameter space: $M_{\star}, T_{\mathrm{eff}}$, and $\log \left(M_{\mathrm{He}} / M_{\star}\right)$. All the combinations of parameters found by the GA algorithm for our best fit model with residuals smaller than $3.3 \mathrm{~s}$ are presented. The two panels are orthogonal projections of the search space, so each point in the left panel corresponds to a point in the right panel. The fit is displayed using the following color scale: a red point if the residuals ( $\mathrm{rms}$ ) are smaller than $2.2 \mathrm{~s}$; yellow: $2.2 \leq \mathrm{rms}<2.4$; green: $2.4 \leq \mathrm{rms}<2.7$; cyan: $2.7 \leq \mathrm{rms}<3.0$; blue: $3.0 \leq \mathrm{rms}<3.3$. The figure shows the presence of more than one region that yields a good match to the observations. Two families of good fits, for thick and thin helium layers, are displayed, but the amount and quality of the fits found for thin layers are larger. The distribution of the best models form stripes in the search spaces panels. This is due to the parameter correlations in both projections. The correlation between $M_{\star}$ and $\log \left(M_{\mathrm{He}} / M_{\star}\right)$ is described by Brassard et al. (1992), who showed that a thinner helium layer can compensate for an overestimate of the mass.

The correlation between $M_{\star}$ and $T_{\text {eff }}$ is related to the BruntVäisälä frequency, which reflects the difference between the actual an the adiabatic density gradients. The pulsation periods of a white dwarf model reflect the average of the Brunt-Väisälä frequency through the star. If the temperature decreases, the matter becomes more degenerate, so the Brunt-Väisälä frequency tends to be zero in much of the star, leading to lower pulsation frequencies. So an overestimate of the mass can compensate for an underestimate of the temperature.

Figure 4 presents the observed and calculated periods for the best model plotted against the forward period spacing $(\Delta P \equiv$ $\left.P_{k+1}-P_{k}\right)$. Note that the GA only fits the periods of the pulsations modes, and the agreement between the deviations from the mean period spacing is a reflection of the overall quality of the match.

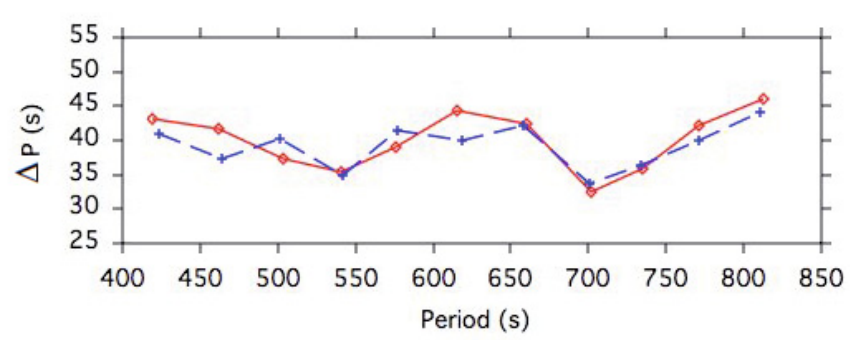

Fig. 4. The observed periods of GD 358 (dashed line), and the optimal model periods found by the genetic algorithm (solid line), using a profile for a remnant of $0.894 M_{\odot}$ from binary evolution with 2 CEs.

\section{Discussion}

We have presented a new method to investigate the evolutionary history of GD 358, which uses the dependence of the pulsation frequencies on the internal $\mathrm{C} / \mathrm{O}$ profile. Different evolutionary channels lead to the generation of different profiles describing how the $\mathrm{C} / \mathrm{O}$ ratio changes from the core of the star to its surface. We have tested and compared profiles associated to binary evolution where the object experiences one or two episodes of mass loss during one or two CE phases. In all the cases the first CE happens during the RGB phase. We have also analyzed two profiles obtained from single evolution channels for comparison.

Our results confirm the dependence of the pulsations on the shape of the $\mathrm{C} / \mathrm{O}$ profile and indicate that binary evolution may provide a better match to the pulsation frequencies of GD 358 . Our best-fit model involves a previous evolutionary history with two CE episodes that has removed all of the hydrogen from the surface of the star. This is an alternative way to explain the lack of hydrogen in DB white dwarfs. IT85 estimated a formation rate of $\sim 10^{-2} \mathrm{yr}^{-1}$ of non-DA degenerate dwarfs created from these binary scenarios, and concluded that they may produce $15 \%-20 \%$ of all non-DA white dwarfs. Our results suggests that 
GD 358 may be one of these. The best-fit model has a mass close to the mean mass for DB white dwarfs, a temperature consistent with recent calibration of UV spectra obtained with the IUE satellite, and a thin outer layer of helium. However, the indications that binary evolution describes better the pulsations pattern showed by GD 358 are based in the analysis of few profiles, and these are related to remnants with different masses. The remnant mass may also affect the profile shape and, therefore, the fit. A larger family of $\mathrm{C} / \mathrm{O}$ profiles of white dwarfs models with masses close to that expected for GD 358, evolved through several binary and single star evolutionary channels, would be necessary to test to place this result on a more solid basis, and to improve a more significant difference in the quality of the period fits.

The value obtained for $M_{\star}$ in our best-fit model $\left(0.585 M_{\odot}\right)$ is lower than the IT85 mass value related to the profile that produced this best-fit $\left(0.894 M_{\odot}\right)$. In general, there are differences between these values for the different profiles tested. This may indicate that the physics of the evolutionary code and the white dwarf models are not completely consistent. However, in most of the cases, we have included profiles that use the same evolutionary code to be able to do qualitative comparisons, avoiding possible differences in the fits due to the different physics applied to the models. Therefore, the method presented may also be used to investigate the coherence between evolutionary codes and the adiabatic non-radial oscillation code. Further $\mathrm{C} / \mathrm{O}$ profiles obtained with improved physics must be analyzed to investigate this relation.

The results obtained for GD 358 indicate that this method may be a good option to investigate the evolution of DBV white dwarfs. This method is strongly related to observational properties of the stars, in particular to their pulsation periods. The main problem is that it can only be applied to few DBV white dwarfs: those that have been extensively observed in multisite campaigns in order to resolve their temporal spectrum. Currently there are only two candidates suitable for this study: GD 358 and CBS 114 (Handler et al. 2002; Metcalfe et al. 2005). Further options for the future include extending this study using profiles coming from double evolutionary scenarios where the star experienced an epoch of mass loss from one $\mathrm{CE}$ episode during the AGB phase.

\section{References}

Althaus, L. G., \& Corsico, A. H. 2003, A\&A, 417, 1115

Beauchamp, A., Wesemael, F., Bergeron, P., \& Liebert, J. 1995, ApJ, 441, 85

Beauchamp, A., Wesemael, F., Bergeron, P., et al. 1999, ApJ, 516, 887
Bradley, P. A. 1993, Ph.D. Thesis, University of Texas-Austin Bradley, P. A., \& Winget, D. E. 1994, ApJ, 430, 850

Brassard, P., Fontaine, G., Wesemael, F., \& Hansen, C. J. 1992, ApJS, 80, 369

Böhm, K. H., \& Cassinelli, J. 1971, A\&A, 12, 21

Castanheira, B. G., Nitta, A., Kepler, S. O., Winget, D. E., \& Koester, D. 2005, A\&A, 432, 175

Castanheira, B. G., Kepler, S. O., Handler, G., \& Koester, D. 2006, A\&A, 450, 331

Corsico, A. H., Althaus, L. G., Kepler, S. O., et al. 2008, A\&A, 478, 869

Eisenstein, D. J., Liebert, J., \& Koester, D. 2006, AJ, 132, 676

Geist, A., Beguelin, A., Dongarra, J., et al. 1994, PVM: Parallel Virtual Machine, A User Guide and Tutorial for Networked Parallel Computing (Cambridge: MIT Press)

Gonzalez Perez, J. M., Solheim, J.-E., \& Kamben, R. 2006, A\&A, 454, 527

Han, Z., Tout, C. A., \& Egglenton, P. P. 2000, MNRAS, 319, 215

Handler, G., Metcalfe, T. S., \& Wood, M. A. 2002, MNRAS, 335, 698

Iben, I. Jr. 1976, ApJ, 208, 165

Iben, I. Jr. 1982, ApJ, 259, 244

Iben, I. Jr. 1986, ApJ, 304, 201 (I86)

Iben, I. Jr., \& Tutukov, A. V. 1985, ApJS, 58, 661 (IT85)

Iglesias, C. A., \& Rogers, F. J. 1996, ApJ, 464, 943

Itoh, N., Hayashi, H., Nishikawa, A., \& Kohyama, Y. 1996, ApJS, 102, 411

Kawaler, S. 1986, Ph.D. Thesis, University of Texas-Austin

Kepler, S. O. 1984, ApJ, 286, 314

Kepler, S. O., Nather, E. N., Winget, D. E., et al. 2003, A\&A, 401, 639

Kepler, S. O., Kleinman, S. J., Nitta, A., et al. 2007, MNRAS, 375, 1315

Kleinman, S. J., Harris, H. C., Eisenstein, D. J., et al. 2004, ApJ, 607, 426

Kutter, G. S., \& Savedoff, M. P. 1969, ApJ, 156, 1021

Lamb, D. Q., \& Van Horn, H. M. 1975, ApJ, 200, 306

Liebert J. 1986, (Dordrecht: D. Reidel Publishing Co.), Proc. IAU Coll., 87367

Metcalfe, T. S., \& Nather, R. E. 1999, Linux J., 65, 58

Metcalfe, T. S., \& Nather, R. E. 2000, Baltic Astron., 9, 479

Metcalfe, T. S., Nather, R. E., \& Winget, D. E. 2000, ApJ, 545, 974 (M0)

Metcalfe, T. S., Winget, D. E., \& Charbonneau, P. 2001, ApJ, 557, 1021 (M1)

Metcalfe, T. S., Nather, R. E., Watson, T. K., et al. 2005, A\&A, 435, 649

Montgomery, M. H. 1998, Ph.D. Thesis, University of Texas-Austin

Montgomery, M. H., Metcalfe, T. S., \& Winget D. E. 2001, ApJ, 548, L53

Montgomery, M. H., Metcalfe, T. S., \& Winget, D. E. 2003, MNRAS, 344, 657

Montgomery, M. H., Williams, K. A., Winget, D. E., et al. 2008, ApJ, 678, L51

Nather, R. E., Robinson, E. L., \& Stover, R. J. 1981, ApJ, 244, 269

Nather, R. E., Winget, D. E., Clemens, J. C., Hansen, C. J., \& Hine, B. P. 1990, ApJ, 361, 309

Salaris, M., Dominguez, I., Garcia-Berro, E., et al. 1997, ApJ, 486, 413

Saumon, D., Chabrier, G., \& van Horn, H. M. 1995, ApJS, 454, 527

Shipman, H. 1997, in White dwarfs: Proc. 10th European Workshop on White Dwarfs (Dordrecht: Kluwer), 165

Robinson, E. L., Kepler, S. O., \& Nather, R. E. 1982, ApJ, 259, 219

Vuille, F., O'Donoghue, D., Buckley, D. A. H., et al. 2000, MNRAS, 314, 689

Winget, D. E. 1981, Ph.D. Thesis, University of Rochester

Winget, D. E., Robinson, E. L., Nather, R. E., et al. 1985, ApJ, 292, 606

Winget D. E., Nather, R. E., Clemens, J. C., et al. 1994, ApJ, 430, 839

Wolff, B., Koester, D., Montgomery, M. H., \& Winget, D. E. 2002, A\&A, 388, 320

Wood, M. 1990, Ph.D. Thesis, University of Texas-Austin 